\title{
Tagged and untagged TRAIL show different activity against tumor cells
}

\author{
KUNPENG ZHAO ${ }^{1,2}$, YAN'GE WANG ${ }^{1,2}$, XUEYIN WANG $^{4}$, YUGANG WANG $^{3}$ and YUANFANG MA ${ }^{1,2}$ \\ ${ }^{1}$ Department of Cell Biology, Institute of Immunology, Medical College of Henan University; ${ }^{2}$ Department of Immunology, \\ Key Laboratory of Cellular and Molecular Immunology, Henan University, Kaifeng, Henan 475003; ${ }^{3}$ Department of \\ Immunology, Institute of Basic Medical Sciences, Beijing 100850; \\ ${ }^{4}$ Department of Immunology, Henan Medical College for Staff and Workers, Zhengzhou, Henan 451191, P.R. China
}

Received May 18, 2012; Accepted August 16, 2012

DOI: $10.3892 / \mathrm{ol} .2012 .908$

\begin{abstract}
The tumor necrosis factor-related apoptosis-inducing ligand (TRAIL/Apo2L) is a novel cytotoxic ligand belonging to the TNF superfamily which is currently being developed as a cancer therapeutic drug. Here, we observed the different functions of recombinant TRAIL protein with a foreign protein label and non-labeled TRAIL. We used a prokaryotic expression system to prepare two different versions of the extracellular TRAIL 114-281aa protein: TRAIL-HS, a protein modified with 6xHis-Tag and S-Tag; and TRAIL-FT, which had no foreign protein. The proteins were purified using Ni-NTA chromatography (TRAIL-HS) and cation ion-exchange column chromatography (TRAIL-FT) and identified by SDS-PAGE and western blot analysis. We compared the abilities of the proteins to bind to death receptor 5 (DR5) by ELISA and to induce apoptosis in a normal liver cell line (Chang liver) and a human T-lymphocyte leukemia cell line (Jurkat) by MTT assay, GR staining and FACS. The results indicate that the biological functions of TRAIL-FT were superior to those of TRAIL-HS in binding and the induction of apoptosis, and may be useful to further the development and applications of TRAIL.
\end{abstract}

\section{Introduction}

Tumor necrosis factor-related apoptosis-inducing ligand (Apo2L/TRAIL), a type II transmembrane protein, is a member of the TNF superfamily (1). In humans, TRAIL binds to two death-inducing receptors, DR4/TRAIL-R1 and

Correspondence to: Dr Yugang Wang, Institute of Basic Medical Sciences, 27 TaiPing Avenue, Beijing 100850, P.R. China

E-mail: wangyg03@163.com

Professor Yuanfang Ma, Institute of Immunology, 88 MingLun Avenue, Medical College of Henan University, Kaifeng, Henan 475003, P.R. China

E-mail: mayf@henu.edu.cn

Key words: recombinant TRAIL, prokaryotic expression, apoptosis
DR5/TRAIL-R2/KILLER. TRAIL has also been shown to bind three decoy receptors, DcR1/TRID/TRAIL-R3, DcR2/TRAIL-R4 and a soluble receptorosteoprotegerin(OPG) that was originally identified as a receptor for OPGL/RANKL. The soluble form of TRAIL exhibits apoptotic activity against various cancer cell lines with minimal cytotoxicity toward normal tissues in vitro and in vivo (2). Notable exceptions are immature human and mouse dendritic cells (DCs) that are sensitive to TRAIL-mediated apoptosis in vitro $(3,4)$. Ligands for the DRs, TNF and FasL, have been shown to induce serious toxic effects following systemic administration $(5,6)$. There is also concern that certain rTRAIL variants may induce systemic toxicity, highlighting the importance of preclinical assessment for this ligand. Indeed, certain types of TRAIL show cytotoxicity to normal cells. Polyhistidinetagged recombinant human TRAIL has been shown to induce apoptosis in normal human hepatocytes (5), recombinant human leucine zipper (LZ)- and polyhistidine-tagged TRAIL have been shown to induce apoptosis in normal keratinocytes $(3,7)$, and recombinant LZ-TRAIL is cytotoxic to human astrocytes in vitro (1). By contrast, other studies have revealed that rTRAIL lacking exogenous sequences does not induce apoptosis in normal human and cynomolgus monkey hepatocytes (6), human mammary, renal or prostatic epithelial cells, umbilical vein endothelial cells, lung fibroblasts, colon smooth muscle cells, astrocytes or keratinocytes (7-9). However, controversy remains concerning which type of TRAIL is superior.

In this study, TRAIL-FT, which comprises TRAIL (114-281aa) without any exogenous sequences, was expressed by a prokaryotic expression system. Its identity was characterized and its functions were analyzed in comparison with those of TRAIL-HS, a tagged form of TRAIL (114-281aa) with a 45 aa exogenous sequence including 6xHis-tag and S-tag.

This study was performed with the approval of the ethical committee of Henan University, Henan, China.

\section{Materials and methods}

Construction and expression of TRAIL-HS and TRAIL-FT. The primers were designed according to the cDNA sequence 


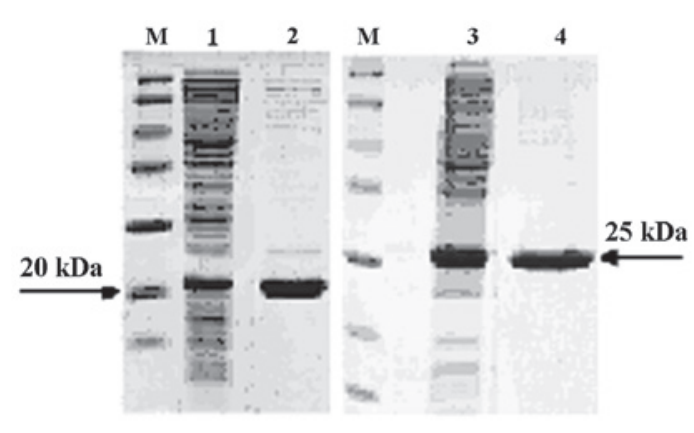

Figure 1. Expression and purification of tumor necrosis factor-related apoptosis-inducing ligand (TRAIL) proteins. M, Marker; lanes 1 and 3 , supernatant; lane 2, purified TRAIL-FT; lane 4, purified TRAIL-HS.

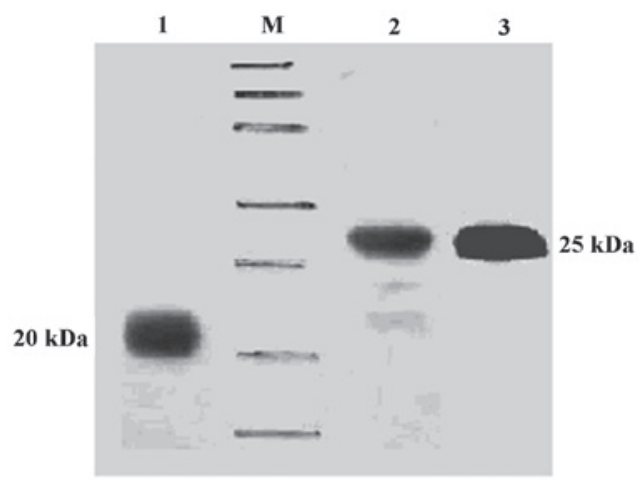

Figure 2. Identification of tumor necrosis factor-related apoptosis-inducing ligand (TRAIL) proteins by western blotting. TRAIL-FT and TRAIL-HS were resolved by SDS-PAGE, transferred to nitrocellulose and incubated with anti-TRAIL polyclonal antibody and anti-His antibody, respectively. Secondary antibody was added and the membrane was washed prior to development with enhanced chemiluminescence (ECL) reagents. M, marker line; lane 1, TRAIL-FT incubated with poly-anti-TRAIL antibody; lanes 2 and 3, TRAIL-HS incubated with anti-His antibody and poly-anti-TRAIL antibody, respectively.

of TRAIL provided in GenBank and synthesized by Invitrogen Biotechnology Co., Ltd. (Shanghai, China). The primers were sense: 5'-CATGCCATGGTGAGAGAAAGAGGTCCT CAG-3', and anti-sense: 5'-TCCGCTCGAGCGGTTAGC CAACTAAC-3'. The underlined sequences are $\mathrm{NcoI}$ and $\mathrm{XhoI}$ sites, respectively. The total RNA of human PBMC was extracted using the TRIzol reagent (Invitrogen Biotechnology Co., Ltd.). The cDNA was synthesized using M-MLV reverse transcriptase (Takara Bio Inc., Shiga, Japan) with total RNA as the template. The TRAIL gene was obtained by PCR amplification, cloned into pGEM-T-easy vector (Promega, Madison, WI, USA) and sequenced. TRAIL extracellular gene was digested from the sequencing vector and ligated to pET-30a and pET-28a prokaryotic expression vectors with T4 ligase (New England BioLabs, Inc., Ipswich, MA, USA). The TRAIL-HS and TRAIL-FT proteins were expressed in E.coli BL21(DE3) induced by IPTG (0.1 mM; Sigma, St. Louis, MO, USA) and purified by Ni-NTA and SP column chromatography, respectively.

Western blotting. The two TRAIL proteins expressed in E.coli BL21(DE3) were resolved by SDS-PAGE on $15 \%$ poly-
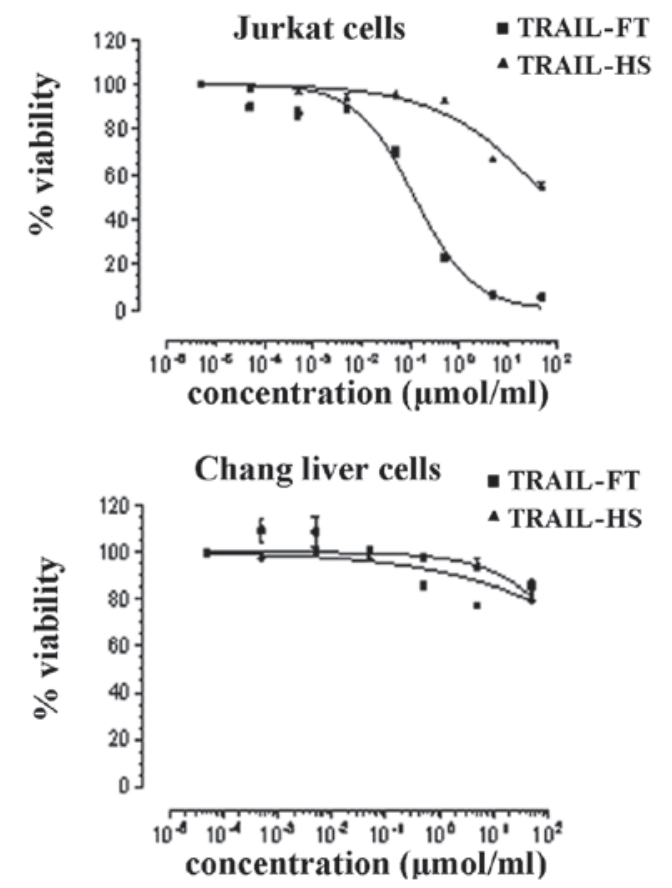

Figure 3. Inhibition of proliferation by tumor necrosis factor-related apoptosis-inducing ligand (TRAIL)-FT and TRAIL-HS. Jurkat cells and Chang liver cells were dispensed into 96-well culture plates. TRAIL-FT and TRAIL-HS proteins were added to each well. After 12 h, 3-(4,5-dimethylthiazol-2-yl)-2,5-diphenyl tetrazolium bromide (MTT) was added. A solubilization solution was added to resolve the formazan salt crystals. The plate was then read at $570 \mathrm{~nm}$ using a plate reader. All experiments were performed in triplicate and repeated at least three times.
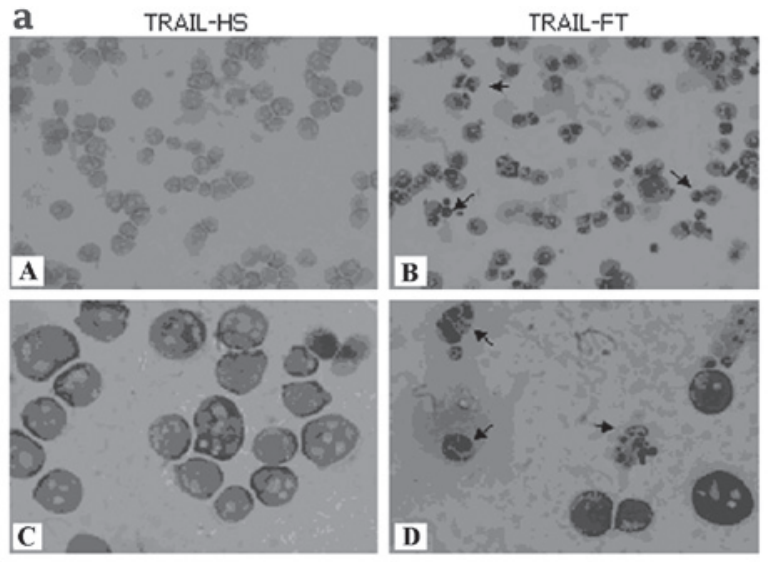

b

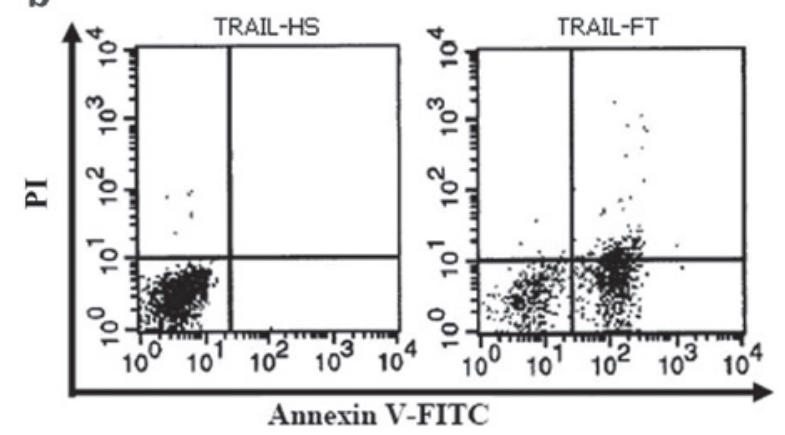

Figure 4. Induction of apoptosis by tumor necrosis factor-related apoptosis-inducing ligand (TRAIL)-HS and TRAIL-FT. Apoptosis of the Jurkat cells was identified by (a) Wright-Giemsa staining and (b) FACS. Jurkat cells incubated with (A and C) TRAIL-HS and (B and D) TRAIL-FT. (C and D) are magnified images of (A and B), respectively. 
acrylamide gels and transferred to a nitrocellulose membrane using a horizontal electrophoresis transfer system (Bio-Rad, Hercules, CA, USA). The membrane was blocked with 5\% non-fat milk for $1 \mathrm{~h}$ and then incubated with poly-anti-TRAIL antibody (eBioscience, San Diego, CA, USA) or anti-His-Tag antibody (Tiangen Biotech, Beijing, China) at room temperature for $1 \mathrm{~h}$. After washing twice with PBST, the membrane was incubated with HRP-conjugated secondary antibody. The blots were developed using enhanced chemiluminesence (ECL) reagents. mDRA6 was used as a positive control.

Proliferation inhibition assay. Jurkat and Chang liver cells (American Type Culture Collection, Manassas, VA, USA) were used to test the antiproliferative activities of the two TRAIL proteins. Briefly, $100 \mu 1$ Jurkat and Chang liver cells $\left(8 \times 10^{5} / \mathrm{ml}\right)$ were dispensed into the wells of 96 -well cell culture plates. The TRAIL-HS and TRAIL-FT proteins were diluted (final concentration: $10^{-5}, 10^{-4}, 10^{-3}, 10^{-2}, 10^{-1}$, $10^{0}, 10^{1}$ or $10^{2} \mathrm{pmol} / \mathrm{ml}$ ) and added to the wells in triplicate. The plates were kept in an incubator at $37^{\circ} \mathrm{C}$ with $5 \% \mathrm{CO}_{2}$ for $12 \mathrm{~h}$. After adding $10 \mu \mathrm{l}$ 3-(4,5-dimethylthiazol-2-yl)2,5-diphenyl tetrazolium bromide (MTT; $10 \mathrm{mg} / \mathrm{ml}$; Sigma), the plates were incubated for an additional $4 \mathrm{~h}$ at $37^{\circ} \mathrm{C}$. Following this incubation, $100 \mu \mathrm{l}$ solubilization solution was added and incubation was continued at $37^{\circ} \mathrm{C}$ for $12 \mathrm{~h}$. The plate was then read at $570 \mathrm{~nm}$ using a plate reader (Anthos Labtec Instruments GmbH, Salzburg, Austria). The following formula was used to estimate the proliferation inhibition rates of TRAIL-HS and TRAIL-FT: Proliferation inhibition rate $(\%)=\left(1-\mathrm{A}_{570 \text { (sample) }}\right) /\left(\mathrm{A}_{570 \text { (control) }}\right) \times 100$.

Detection of cell apoptosis. Two experiments were carried out to detect the apoptosis induced by the two recombinant TRAIL proteins. The Jurkat cells were treated with TRAIL-HS or TRAIL-FT (20 ng/ml) for $100 \mathrm{~min}$ and then washed once with PBS. PBS was used as the reagent control. Each sample was then divided into two equal portions. One portion was treated with $75 \%$ ethanol at $-20^{\circ} \mathrm{C}$ for $24 \mathrm{~h}$ and then incubated with $50 \mathrm{mg} / \mathrm{ml} \mathrm{RNase} A$ at $37^{\circ} \mathrm{C}$ for $30 \mathrm{~min}$. PI $(50 \mathrm{mg} / \mathrm{ml})$ was used to stain the cells for $30 \mathrm{~min}$ and the cells were analyzed by flow cytometry (FACSCalibur; Becton-Dickinson, Franklin Lakes, NJ, USA) using the software program CELLQUEST ${ }^{\mathrm{TM}}$. Ten thousand events were counted. The other portion of the sample was distributed on a slide with a cell centrifuge, fixed with methanol for $5 \mathrm{~min}$ and then stained with Wright-Giemsa staining buffer. The slides were observed under an immersion objective lens and images captured to enable the identification of the apoptosis induced by the proteins from the cell morphology.

\section{Results}

Expression and purification of TRAIL-FT and TRAIL-HS proteins. The two TRAIL proteins were purified using Ni-NTA and SP chromatography columns. The results revealed that the target proteins existed in the supernatants of the lysed E.coli BL21(DE3). High purity proteins were obtained (Fig. 1). Western blot analysis indicated positive reactions for TRAIL-FT and TRAIL-HS with poly-anti-TRAIL and anti-His-Tag antibodies (Fig. 2).
Inhibition of cell proliferation by TRAIL-HS and TRAIL-FT proteins. The two proteins inhibited the proliferation of Jurkat cells significantly at concentrations of $10^{-4}-10^{2} \mathrm{nmol} / \mathrm{ml}$. The performance of the TRAIL-FT protein is notable. Furthermore, when incubated with Chang liver cells, the proteins revealed little or no cytotoxicity (Fig. 3).

Apoptosis induced by TRAIL-FT and TRAIL-HS. The results of Wright-Giemsa staining (Fig. 4a) revealed that the Jurkat cells incubated with TRAIL-FT acquired the typical features of apoptosis, including cell shrinkage, membrane blebbing and nuclear pyknosis, unlike those incubated with TRAIL-HS. The results of FACS analysis (Fig. 4b) indicate that TRAIL-FT is more potent than TRAIL-HS in the induction of apoptosis.

\section{Discussion}

TRAIL has anticancer activity when used as a single agent or in combination with chemotherapeutic agents. TRAIL induces apoptosis by interacting with death receptors (DR4,DR5). When TRAIL binds to DR4 and/or DR5, the receptors become trimerized to form the death-inducing signaling complex (DISC) and recruit the adapter protein FADD and caspase- 8 or caspase-10. This leads to activation of the executioner caspases, including caspase-3, and cleavage of the death substrates to cause cell death (10). An earlier study indicated that a polyhistidine-tagged version of rhTRAIL caused apoptosis in cultured human and nonhuman primate hepatocytes, which raised concerns about its safety (11). Further studies suggested that hepatocyte toxicity caused by polyhistidine-tagged TRAIL is attributable to low levels of zinc $(12,13)$. In our study, high-level expression of soluble fusion proteins (TRAIL-FT and TRAIL-HS) were achieved in E.coli BL21(DE3). Large amounts of pure and active rhTRAIL proteins were purified by Ni-NTA and cation ion-exchange chromatography. By structural analysis, we conclude that the tag protein resulted in the formation of polymeric TRAIL and affected its functions. Our study revealed that recombinant non-tagged TRAIL protein is noteworthy as a potential anticancer drug. However, further investigation of the mechanism of TRAIL-induced apoptosis is required.

\section{Acknowledgements}

This study was supported by the National Major Scientific and Technological Special Project for 'Significant New Drugs Creation' (2011ZX09506-004).

\section{References}

1. Wiley SR, Schooley K, Smolak PJ, et al: Identification and characterization of a new member of the TNF family that induces apoptosis. Immunity 3: 673-682, 1995.

2. Walczak H, Miller RE, Ariail K, et al: Tumoricidal activity of tumor necrosis factor-related apoptosis-inducing ligand in vivo. Nat Med 5: 157-163, 1999.

3. Leverkus M,Walczak H,McLellan A,etal: Maturation of dendritic cells leads to up-regulation of cellular FLICE-inhibitory protein and concomitant down-regulation of death ligand-mediated apoptosis. Blood 96: 2628-2631, 2000.

4. Yagita H, Takeda K, Hayakawa Y, et al: TRAIL and its receptors as targets for cancer therapy. Cancer Sci 95: 777-783, 2004. 
5. Jo M, Kim TH, Seol DW, Esplen JE, et al: Apoptosis induced in normal human hepatocytes by tumor necrosis factor-related apoptosis-inducing ligand. Nat Med 6: 564-567, 2000.

6. Lawrence D, Shahrokh Z, Marsters S, et al: Differential hepatocyte toxicity of recombinant Apo2L/TRAIL versions. Nat Med 7: 383-385, 2001.

7. Ashkenazi A, Pai RC, Fong S, et al: Safety and antitumor activity of recombinant soluble Apo2 ligand. J Clin Invest 104: 155-162, 1999.

8. Cretney E, Shanker A, Yagita H, et al: TNF-related apoptosis-inducing ligand as a therapeutic agent in autoimmunity and cancer. Immunol Cell Biol 84: 87-98, 2006.

9. Qin JZ, Bacon P, Chaturvedi V and Nickoloff BJ: Role of NF- $\kappa B$ activity in apoptotic response of keratinocytes mediated by interferon-gamma, tumor necrosis factor- $\alpha$, and tumor necrosis factor-related apoptosis-inducing ligand. J Invest Dermatol 117: 898-907, 2001.
10. Wu GS: TRAIL as a target in anti-cancer therapy. Cancer Lett 285: 1-5, 2009.

11. Jo M, Kim TH, Seol DW, et al: Apoptosis induced in normal human hepatocytes by tumor necrosis factor-related apoptosis-inducing ligand. Nat Med 6: 564-567, 2000.

12. Lawrence D, Shahrokh Z, Marsters S, et al: Differential hepatocyte toxicity of recombinant Apo2L/TRAIL versions. Nat Med 7: 383-385, 2001.

13. Sun AY, Shen YL, Yin JC, et al: Improvement of expression level and bioactivity of soluble tumor necrosis factor-related apoptosis-inducing ligand (Apo2L/TRAIL) by a novel zinc ion feeding strategy. Biotechnol Lett 28: 1215-1219, 2006. 